Also available at http://amc.imfm.si

ISSN 1855-3966 (printed edn.), ISSN 1855-3974 (electronic edn.)

ARS MATHEMATICA CONTEMPORANEA 6 (2013) 127-145

\title{
Consensus strategies for signed profiles on graphs
}

\author{
Kannan Balakrishnan \\ Department of Computer Applications, Cochin University of Science and Technology \\ Cochin - 682 022, India \\ Manoj Changat * \\ Department of Futures Studies, University of Kerala, Trivandrum - 695 034, India \\ Henry Martyn Mulder ${ }^{\dagger}$ \\ Econometrisch Instituut, Erasmus Universiteit \\ P. O. Box 1738, 3000 DR Rotterdam, Netherlands
}

Ajitha R. Subhamathi

Department of Computer Applications, N.S.S. College, Rajakumari, Idukki, Kerala, India

Received 18 October 2011, accepted 28 March 2012, published online 15 June 2012

\begin{abstract}
The median problem is a classical problem in Location Theory: one searches for a location that minimizes the average distance to the sites of the clients. This is for desired facilities as a distribution center for a set of warehouses. More recently, for obnoxious facilities, the antimedian was studied. Here one maximizes the average distance to the clients. In this paper the mixed case is studied. Clients are represented by a profile, which is a sequence of vertices with repetitions allowed. In a signed profile each element is provided with a sign from $\{+,-\}$. Thus one can take into account whether the client prefers the facility (with a + sign) or rejects it (with a - sign). The graphs for which all median sets, or all antimedian sets, are connected are characterized. Various consensus strategies for signed profiles are studied, amongst which Majority, Plurality and Scarcity. Hypercubes are the only graphs on which Majority produces the median set for all signed profiles. Finally, the antimedian sets are found by the Scarcity Strategy on e.g. Hamming graphs, Johnson graphs and halfcubes.
\end{abstract}

Keywords: Plurality strategy, median, majority rule, Hamming graph, Johnson graph, halfcube.

Math. Subj. Class.: 05C99, 05C12, $90 B 80$

\footnotetext{
${ }^{*}$ Research work is supported by NBHM/DAE under grant No.2/48(2)/2010/NBHM-R \& D

$\dagger$ Research work was carried out when this author visited University of Kerala, under the Erudite Scheme of the Government of Kerala, January 3-16, 2011

E-mail addresses: bkannan@cusat.ac.in (Kannan Balakrishnan), mchangat@gmail.com (Manoj Changat), hmmulder@few.eur.nl (Henry Martyn Mulder), ar.subhamathi@gmail.com (Ajitha R. Subhamathi)
} 


\section{Introduction}

Most of the facility location problems in the literature are concerned with finding locations for desirable facilities. The goal there is to minimize a distance function between facilities and the demand sites (clients). One way to model this is using a network, see for instance $[23,24,17]$. In the discrete case one uses graphs, and clients and facilities are to be positioned on vertices.

One may formulate such location problems also in terms of achieving a consensus amongst the clients. Thus it becomes a problem in Consensus Theory. This approach has been fruitful in many other applications, e.g. in social choice theory, clustering, and mathematical biology, see for instance [7, 16, 15, 22].

From the view point of median consensus the classical result of Goldman [12] is very interesting: to find the median in a tree, just move to the majority of the clients. In [20], this majority strategy was formulated for arbitrary graphs. It was proved that majority strategy finds all medians for any set of clients if and only if the graph is a so-called median graph. Clients are termed as profiles in the language of graph theory, defined as a sequence of vertices in which vertices are allowed to repeat.

The class of median graphs comprises that of the trees as well as that of the hypercubes and grids. It allows a rich structure theory $[18,13,21]$ and has many and diverse applications, see, for. e.g., [14], for median type consensus. In the majority strategy we compare the two ends of an edge $v$ and $w$ : if we are at $v$ and at least half of the clients are strictly nearer to $w$ than to $v$, then we move to $w$. One could relax the requirement for making a move as follows: one may move to $w$ if there are at least as many clients closer to $w$ than to $v$. Note that in the latter case less than half may actually be closer to $w$ because there are many clients having equal distance to $v$ and $w$. This idea of relaxing the majority strategy is formalized as plurality strategy in [4]. Other consensus strategies known as Condorcet, hill climbing and Steepest ascent hill climbing strategies were also proposed in [4]. There it is proved that the plurality, hill climbing and steepest ascent hill climbing strategies starting at an arbitrary vertex for arbitrary profiles will always return the median set of the profile if and only if the graph has connected medians.

However just as important are the problems dealing with the location of undesirable or obnoxious facilities, such as nuclear reactors, garbage dumps or water purification plants, see $[9,10,11]$. Here the criterion for optimality is maximizing the sum of the distances from the location of the obnoxious facility to the locations of the clients. The problem is known as the antimedian problem.

In general any two subgraphs may appear as antimedian and median sets, respectively, for clients located at all vertices without repetitions, with the distance between them being arbitrary, see for instance [2]. It is possible that facilities which are undesirable for some clients may be desirable for some other clients. For example, assume the problem of locating a beer parlour in a human habitat area. Some of the inhabitants may consider it as a desirable facility where as some others may consider it as undesirable facility. One way to formulate such problem is to associate a sign with the clients indicating whether the facility is desirable or undesirable to the client. In this paper we are concentrating on methods to solve such problems. For this a more general concept called signed profiles is introduced and is formally defined in the next section. In Section 3, the equivalence of rational weight functions and signed profiles are established, and the relationship between the median and antimedian sets for signed profiles is obtained. In Section 4, various consensus strategies are formulated, amongst which Majority, Plurality and Scarcity strategy, and it is shown 
that all these consensus strategies are pairwise distinct for signed profiles, as it has already been known for the usual profiles. We show that, for signed profiles, the hypercubes are the only graphs on which Majority produces the median set for any signed profile in Section 5. Finally, for Scarcity, we study various classes of graphs, on which this strategy produces the antimedian set for any signed profile.

\section{Preliminaries}

Let $G=(V, E)$ be a finite, connected, simple graph with vertex set $V$ and edge set $E$. The distance function of $G$ is denoted by $d$, where $d(u, v)$ is the length of a shortest $u, v$-path. We call a subset $W$ of $V$ connected if it induces a connected subgraph in $G$. The interval $I(u, v)$ between two vertices $u$ and $v$ consists of all vertices on shortest $u, v$-paths, that is:

$$
I(u, v)=\{x \mid d(u, x)+d(x, v)=d(u, v)\} .
$$

A profile on $G$ is a finite sequence $\pi=\left(x_{1}, x_{2}, \ldots, x_{k}\right)$ of vertices of $G$. The length of $\pi$ is the number $k=|\pi|$. Note that, $\pi$ being a sequence, multiple occurrences are allowed. In this paper we extend the concept of profile: a signed profile is a profile where a sign from $\{+,-\}$ is added to each element. We write the sign of element $x_{i}$ as $s_{i}$. Thus a signed profile is a sequence $\pi=\left(s_{1} x_{1}, s_{2} x_{2}, \ldots, s_{k} x_{k}\right)$. We call $x_{i}$ an element of $\pi$ and $s_{i}$ its sign. Note that with this usage, a vertex occurring $k$ times in a profile occurs as $k$ different elements in a profile. For an element $x$ of $\pi$ we denote its sign also by $s_{x}$. For computational reasons, we identify a sign $s$ also with the number $s 1=+1$ or -1 , and talk about +1 or -1 as a sign. Thus we can take the sum of signs. As we will see below, a signed profile with all signs being +1 , plays the role of the usual profile without signs. We call such a profile a positive profile. If all signs are -1 , then the profile is negative. Since all our profiles are signed, we call a signed profile just a profile, and omit the adjective 'signed', except in the statements of lemmas and theorems (to avoid confusion with similar lemmas and theorems in the literature). A profile obtained from $\pi$ by changing each $s_{i}$ by $-s_{i}$ is denoted by $-\pi$. The size of a profile $\pi$ is defined as

$$
\|\pi\|=\sum_{i=1}^{k} s_{i} .
$$

So, for positive profiles we have $\|\pi\|=|\pi|$, and for negative profiles we have $\|\pi\|=-|\pi|$.

For an edge $u v$ in $G$, we denote by $\pi_{u v}$ the subprofile of $\pi$ consisting of the elements of $\pi$ strictly closer to $u$ than to $v$, and by $\pi_{u}^{v}$ the subprofile of all elements at equal distance form $u$ and $v$. Note that a profile, by definition, has a positive length. However, for subprofiles we allow the empty subprofile. For instance, a graph is bipartite if and only if the subprofile $\pi_{u}^{v}$ is empty for any edge $u v$ and any profile $\pi$.

In the literature we find such concepts as remoteness, median and antimedian of positive profiles, for e.g., see, [14] and [20]. These are all very natural and the definitions are in accordance with our intuition. Because the definitions for signed profiles are basically the same, we use the same terminology here.

The remoteness of a vertex $v$ to a profile $\pi$ is defined as

$$
D(v, \pi)=\sum_{i=1}^{k} s_{i} d\left(x_{i}, v\right) .
$$


A permutation of the elements in a profile does not change remoteness. Because we are only interested in the remoteness to profiles, we will consider two profiles as the same if they can be obtained from each other by permuting the elements. We write the concatenation of two profiles $\pi$ and $\rho$ as $\pi \rho$. Thus, for any edge $u v$, we can write $\pi=\pi_{u v} \pi_{u}^{v} \pi_{v u}$.

A vertex minimizing $D(v, \pi)$ is called a median of the profile. The set of all medians of $\pi$ is the median set of $\pi$ and is denoted by $M(\pi)$. A vertex maximizing $D(v, \pi)$ is called an antimedian of the profile. The set of all antimedians of $\pi$ is the antimedian set of $\pi$ and is denoted by $A M(\pi)$. The reader has to keep in mind that the effects of the signs might just be contra-intuitive. For instance, if $\pi$ is a negative profile, then a median of $\pi$ is an antimedian of the positive profile $-\pi$.

A vertex $x$ such that $D(x, \pi) \leq D(y, \pi)$, for all neighbors $y$ of $x$, is a local median of $\pi$. The set of all local medians is denoted by $M_{l o c}(\pi)$. If $D(x, \pi) \geq D(y, \pi)$, for all neighbors $y$ of $x$, then $x$ is a local antimedian of $\pi$. The set of all local antimedians is denoted by $A M_{l o c}(\pi)$.

Let $\pi=\left(s_{1} x_{1}, s_{2} x_{2}, \ldots, s_{k} x_{k}\right)$ be a profile, then we have

$$
D(v,-\pi)=\sum_{i=1}^{k}-s_{i} d\left(x_{i}, v\right)=-\sum_{i=1}^{k} s_{i} d\left(x_{i}, v\right)=-D(v, \pi) .
$$

From this observation we deduce that, by replacing a profile $\pi$ by its opposite $-\pi$, the roles of (local) medians and (local) antimedians are exchanged. So we have $M(\pi)=A M(-\pi)$, etcetera. We single out one fact that we need in the sequel.

When the profile is of the form $(-x,+x)$ the median set is equal to the antimedian set and is the entire vertex set of the graph. But this is not the only case when the median and antimedian set are equal. For example, consider a positive profile $\pi$ on a hypercube containing each vertex once. In this case the remoteness is constant and hence the median and antimedian set are the same and coincide with the entire vertex set of the graph. It can also be noted that the situation is the same for $-\pi$. In general, for such positive and negative profiles on so called distance balanced graphs both the median sets and antimedian sets coincide. The case for such positive profiles on the class of distance balanced graphs is proved in [5]. The same situation holds for some special even profiles (both positive and negative) in some other class of graphs, see for instance [1].

Lemma 2.1. Let $G$ be a connected graph and $\pi$ a signed profile on $G$. Then, for any two adjacent vertices $u, v$ in $G$,

$$
\left\|\pi_{u v}\right\| \leq\left\|\pi_{v u}\right\| \text { if and only if } D(u, \pi) \geq D(v, \pi) .
$$

Proof. Since $u v$ is an edge in $G$, we can ignore $\pi_{u}^{v}$ in the following computation.

$$
\begin{aligned}
D(u, \pi)- & D(v, \pi)= \\
= & \sum_{x \in \pi_{u v}} s_{x} d(u, x)+\sum_{x \in \pi_{v u}} s_{x} d(u, x)-\sum_{x \in \pi_{u v}} s_{x} d(v, x)-\sum_{x \in \pi_{v u}} s_{x} d(v, x) \\
= & \sum_{x \in \pi_{u v}} s_{x} d(u, x)+\sum_{x \in \pi_{v u}} s_{x} d(u, x)-\sum_{x \in \pi_{u v}} s_{x}(d(u, x)+1)- \\
& \sum_{x \in \pi_{v u}} s_{x}(d(u, x)-1) \\
= & \left\|\pi_{v u}\right\|-\left\|\pi_{u v}\right\| .
\end{aligned}
$$


From this the assertion follows immediately.

\section{Remoteness with respect to arbitrary weight functions}

The concept of remoteness function and hence of medians and antimedians can also be studied with respect to weight functions defined on the vertex set of a graph. This was studied by Bandelt and Chepoi in [6] for non-negative weight functions in the case of medians. The equivalence of non-negative weight functions and positive profiles and hence the corresponding equivalence of the remoteness function and medians of non-negative weight functions and positive profiles are established in [4].

In this section, we establish that the same conclusion follows for arbitrary weight functions and signed profiles.

A weight function on $G$ is a mapping $f$ from $V$ to the set of real numbers. Note that we now allow negative weights. We say that $f$ has a local minimum at $x \in V$ if $f(x) \leq f(y)$, for every $y$ adjacent to $x$. It has a local maximum if $f(x) \geq f(y)$, for every $y$ adjacent to $x$. The remoteness function with respect to the weight function $f$ is the function $D_{f}$ from $V$ to the set of real numbers defined as:

$$
D_{f}(v)=D(v, f)=\sum_{x \in V} d(v, x) f(x) .
$$

Note that $D_{f}$ is a weight function on $G$ as well. A local median of $f$ is a vertex $u$ such that $D_{f}$ has a local minimum at $u$. A local antimedian is a vertex at which $D_{f}$ attains a maximum. The set of all local medians of a weight function $f$ is denoted by $M_{l o c}(f)$. The set of all local antimedians is denoted by $A M_{l o c}(f)$. A median of $f$ is a vertex $u$ such that $D_{f}$ has a global minimum at $u$. Similarly, an antimedian of $f$ is a vertex at which $D_{f}$ attains a maximum. The median set $M(f)$ of $f$ is the set of all medians of $f$. The antimedian set $A M(f)$ of $f$ is the set of all anti-medians of $f$.

Let $f$ be a weight function on a graph $G$ and let $-f$ be the weight function defined in the obvious way: its value at $x$ is $-f(x)$. Then clearly, we have $D(v, f)=-D(v,-f)$, for any vertex $v$ in $G$. In the sequel we make use of the following obvious facts.

Remark 3.1. Let $f$ be an arbitrary weight function defined on the vertex set of a graph $G$. Then replacing $f$ with $-f$ interchanges the roles of local maxima (minima) of $f$ with local minima (maxima) of $-f$, and hence also interchanges the roles of both local and global medians (antimedians) of $f$ with local and global antimedians (medians) of $-f$, respectively.

Let $\pi$ be a profile on $G$. Then the weight function associated with $\pi$ is the function $f_{\pi}$ with $f_{\pi}(x)=\sum s_{i}$, where the summation is taken over the occurrences of vertex $x$. If $x$ does not occur in $\pi$, then we set $f(x)=0$. The following lemma follows immediately from the definitions. Note that, for any integer-valued weight function $f$, there are infinitely many profiles having $f$ as their associated weight function.

Lemma 3.2. Let $G$ be a connected graph, and let $\pi$ be a signed profile with associated weight function $f_{\pi}$. Then $D(v, \pi)=D\left(v, f_{\pi}\right)$, and hence $M\left(f_{\pi}\right)=M(\pi)$, and $A M\left(f_{\pi}\right)=A M(\pi)$, and $M_{l o c}\left(f_{\pi}\right)=M_{l o c}(\pi)$, and $A M_{l o c}\left(f_{\pi}\right)=A M_{l o c}(\pi)$, for every $v$ in $V$.

Let $f$ be a weight function on a connected graph $G$. For a positive real number $t$, we define $t f$ to be the weight function with $(t f)(x)=t \times f(x)$. Then we have $M(t f)=$ 
$M(f)$ and $M_{l o c}(t f)=M_{l o c}(f)$. Also we have $A M(t f)=A M(f)$ and $A M_{l o c}(t f)=$ $A M_{l o c}(f)$. Finally, $D_{t f}$ has a strict local minimum (maximum) at a vertex $u$ if and only if $D_{f}$ has a strict local minimum (maximum) at $u$. The following lemma is obvious.

Lemma 3.3. Let $g$ be rational weight function on a connected graph $G$. Then there is a signed profile $\pi$ on $G$ such that $f_{\pi}=t g$ for some positive integer $t$.

In other words, antimedians (medians) of signed profiles are exactly antimedians (medians) of rational weight functions. The same holds for local antimedians (medians). Next we show that real-valued weight functions may be replaced by rational-valued weight functions, and thus by profiles, when one wants to compute antimedian (median) sets. We only present the proofs for the antimedian case. This is the one that we need in Sections 4 and 5. The case for the median sets is similar to that in [4], except that one has to take into account the signs. The next two Lemma's are the signed version of Lemma's 5 and 6 in [4]. The proofs are easy adaptations of those in [4]. Because they are short and prepare the way for Proposition 3.6, we include the proofs of the signed versions.

Lemma 3.4. Let $G$ be a connected graph, and let $f$ be a weight function on $G$ such that $D_{f}$ has a local maximum at vertex $u$, which is not a global maximum. Then there is a weight function $g$ such that $D_{g}$ has a strict local maximum at $u$, which is not a global maximum. Furthermore if $f$ is rational, then $g$ may also be taken as a rational function.

Proof. First note that, for any two vertices $x$ and $y$, we have $d(x, y)<n=|V|$. Let $D(u, f)=\epsilon_{1}$. Let $D_{f}$ have a global maximum at $z$, that is, $D(z, f)=\epsilon>\epsilon_{1}$. Let $\epsilon_{2}=\epsilon-\epsilon_{1}$. Now define the function $g$ as follows.

$$
g(v)=\left\{\begin{array}{lll}
f(v) & \text { if } & v \neq u \\
f(u)-\frac{\epsilon_{2}}{n} & \text { if } & v=u
\end{array}\right.
$$

Then $D(u, g)=D(u, f)$, because in these sums the values $f(u)$ and $g(u)$ of the functions at $u$ are multiplied by $d(u, u)=0$. For any vertex $v$ adjacent to $u$, we have

$$
D(v, g)=D(v, f)-\frac{\epsilon_{2}}{n}<D(v, f) \leq D(u, f)=D(u, g) .
$$

So $D_{g}$ has a strict local maximum at $u$. Furthermore,

$$
D(z, g)=D(z, f)-d(u, z) \frac{\epsilon_{2}}{n}>D(z, f)-\epsilon_{2}=D(u, f)=D(u, g) .
$$

So $g$ has a strict local maximum at $u$ that is not a global maximum. Also if $f$ is rational, then $\epsilon_{2}$ is rational. So $g$ is also rational.

Lemma 3.5. Let $G$ be a connected graph with the property that, for each rational weight function $g$, every local maximum of $D_{g}$ is also a global maximum. Then the same property holds for any real-valued weight function $f$ on $G$.

Proof. Assume that for some real-valued weight function $f$ there is a local maximum for $D_{f}$, at some vertex $u$ that is not a global maximum. In view of the preceding lemma, we may assume that $D_{f}$ has a strict local maximum at $u$. Let $D_{f}$ have a global maximum at $z$, and let

$$
\epsilon_{1}=\min \{D(u, f)-D(x, f) \mid x \text { adjacent to } u\}, \epsilon_{2}=D(z, f)-D(u, f),
$$




$$
\epsilon=\frac{\min \left(\epsilon_{1}, \epsilon_{2}\right)}{n^{2}} .
$$

Now choose a rational weight function $g$ such that $g(v)<f(v)$ and $f(v)-g(v)<\epsilon$, for all $v$. Then, for any vertex $x$ adjacent to $u$, we have $D(u, g)>D(u, f)-\epsilon \times n^{2} \geq$ $D(u, f)-\epsilon_{1} \geq D(x, f)>D(x, g)$. So $u$ is a local maximum for $D_{g}$. Moreover, we have $D(z, g)>D(z, f)-\epsilon \times n^{2} \geq D(z, f)-\epsilon_{2} \geq D(u, f)>D(u, g)$. So $u$ is not a global maximum for $D_{g}$, which is a contradiction.

Graphs with connected median sets for non-negative weight functions were characterized in [6]. Using an analogous approach, we now are able to characterize graphs with connected antimedian and median sets for arbitrary weight functions. Before stating the result, we define basic concepts used in the following lines. A subgraph $G$ of a graph $H$ is an isometric subgraph if $d_{G}(u, v)=d_{H}(u, v)$ for all vertices $u, v$ in $G$. We call a subset $S$ of the vertex set of $G$ a level set with respect to an integer $\lambda$ if $S=\left\{x \in V(G): D_{f}(x) \geq \lambda\right\}$.

Proposition 3.6. For a graph $G$ and any arbitrary weight function defined on the vertex set of $G$ the following conditions are equivalent

(i) $A M_{l o c}(f)=A M(f)$ for all weight functions $f$;

(ii) all level sets $\left\{x: D_{f}(x) \geq \lambda\right\}$ induce isometric subgraphs;

(iii) all antimedian sets $A M(f)$ induce isometric subgraphs;

(iv) all antimedian sets $A M(f)$ are connected.

Proof. The implications $(i i) \Rightarrow($ iii $),($ iii $) \Rightarrow(i v)$ are trivial.

Next we prove $(i v) \Rightarrow(i)$. Let $f$ be a weight function. Assume to the contrary that there exists a local antimedian $z$ of $f$ that is not an antimedian. Let $y$ be an antimedian. Amongst such pairs $y, z$, we may choose $y$ and $z$ such that $d(y, z)$ is as small as possible. Our aim is to find two vertices $u$ and $v$ with $d(u, v)=2$ and a weight function $f^{\prime}$ such that $A M\left(f^{\prime}\right)=\{u, v\}$. So $f^{\prime}$ does not have a connected antimedian set.

Consider the interval $I(y, z)$. Because of the minimality of $d(y, z)$, we have $D_{f}(y)>$ $D_{f}(x)$ for all $x$ in $I(y, z)$ distinct from $y$. Since $z$ is a local antimedian, we have $D_{f}(z) \geq$ $D_{f}(x)$, for any neighbor $x$ of $z$, in particular for any neighbor $x$ of $z$ in $I(y, z)$. This implies that $d(y, z) \geq 2$. Hence, going from $y$ to $z$ within $I(y, z)$, we will encounter two vertices $u, v$ such that $d(y, u)=d(y, v)-2, d(z, u)=d(z, v)+2, d(u, v)=2$, with the properties that $D_{f}(u)>D_{f}(x)$ and $D_{f}(v) \geq D_{f}(x)$, for any common neighbor $x$ of $u$ and $v$. Note that these common neighbors of $u$ and $v$ are precisely the vertices in $I(u, v)$ distinct from $u$ and $v$.

If there is any common neighbor $x$ of $u$ and $v$ such that $D_{f}(v)=D_{f}(x)$, then we have $D_{f}(y) \geq D_{f}(u)>D_{f}(v)$. If $D_{f}(v)>D_{f}(x)$ for all common neighbors of $u$ and $v$, then we compare $D_{f}(u)$ and $D_{f}(v)$. If $D_{f}(u) \geq D_{f}(v)$, then again we have $D_{f}(y)>D_{f}(v)$. If $D_{f}(v)>D_{f}(u)$, then we have $D_{f}(y)>D_{f}(v)>D_{f}(u)$. In this case we interchange the names of $u$ and $v$. In all cases we end up with two vertices $u$ and $v$ at distance 2 with

$$
D_{f}(y) \geq D_{f}(u) \geq D_{f}(v) \geq D_{f}(x),
$$

for all common neighbors $x$ of $u$ and $v$, such that, additionally,

$$
D_{f}(y)>D_{f}(v) \text { and } D_{f}(u)>D_{f}(x)
$$

for all common neighbors $x$ of $u$ and $v$. 
We set $\mu_{1}=\frac{D_{f}(u)-D_{f}(v)}{2}$. So $\mu_{1} \geq 0$, and $D_{f}(v)=D_{f}(u)-2 \mu_{1}$. We set $\mu_{2}=$ $D_{f}(y)-D_{f}(v)$. Then $\mu_{2} \geq \mu_{1}$ and $\mu_{2}>0$. Note that for any $x$ in $V$, we have

$$
D_{f}(v) \geq D_{f}(x)-\mu_{2} .
$$

We construct the new weight function $f^{\prime}$ from $f$ as follows

$$
f^{\prime}(x)= \begin{cases}f(x)-\left(\mu_{1}+\mu_{2}\right) & \text { if } x=v \\ f(x)-\left(\mu_{2}\right) & \text { if } x=u \\ f(x) & \text { otherwise. }\end{cases}
$$

Straightforward computation now yields

$$
\begin{aligned}
D_{f^{\prime}}(u) & =D_{f}(u)-2\left(\mu_{1}+\mu_{2}\right) \\
& =D_{f}(v)-2 \mu_{2} \\
& =D_{f^{\prime}}(v) ;
\end{aligned}
$$

and for any vertex $x$ in $I(u, v)$ distinct from $u$ and $v$ :

$$
D_{f^{\prime}}(x)=D_{f}(x)-\mu_{1}-2 \mu_{2}<D_{f}(u)-\mu_{1}-2 \mu_{2}=D_{f^{\prime}}(u)-\mu_{2}<D_{f^{\prime}}(u) ;
$$

and for any vertex $x$ outside the interval (recall that $\mu_{1} \leq \mu_{2}$ ):

$$
D_{f^{\prime}}(x) \leq D_{f}(x)-3 \mu_{2}-\mu_{1} \leq D_{f}(u)-2 \mu_{2}-\mu_{1}<D_{f^{\prime}}(u) .
$$

Thus $A M\left(f^{\prime}\right)=\{u, v\}$, and hence the antimedian set of $f^{\prime}$ is not connected. This impossibility proves this implication.

It remains to prove that $(i) \Rightarrow(i i)$. Let $A M_{l o c}(f)=A M(f)$ for all weight functions $f$. Assume to the contrary that the level set $S=\left\{x \mid D_{f}(x) \geq \lambda\right\}$ corresponding to $\lambda$ is not isometric. Hence there exist two vertices $u, v$ such that no shortest $u, v$-path lies completely inside $S$. Obviously, we can select $u, v$ in $S$ such that $D_{f}(x)<\lambda$ for any $x$ in $I(u, v)$, distinct from $u$ and $v$. Without loss of generality we may assume $D_{f}(u) \leq D_{f}(v)$. Set $D_{f}(z)-D_{f}(u)=\mu_{1}$, where $z$ is an antimedian, and set $\epsilon=\min \left\{D_{f}(u)-D_{f}(w) \mid w \epsilon\right.$ $I(u, v)\}$. Note that, since $D_{f}(u)>\lambda>D_{f}(w)$, for $w$ in $I(u, v)$, we have $\epsilon>0$. Let $\mu_{2}=\frac{\epsilon}{d(u, v)}$. Define a weight function $f^{\prime}$ such that

$$
f^{\prime}(x)= \begin{cases}f(x)-\mu_{1} & \text { if } x=u \\ f(x)-\left(\mu_{1}+\mu_{2}\right) & \text { if } x=v \\ f(x) & \text { otherwise. }\end{cases}
$$

Straightforward computation now yields

$$
\begin{aligned}
D_{f^{\prime}}(v) & =D_{f}(v)-d(u, v) \mu_{1} \\
& >D_{f}(u)-d(u, v)\left(\mu_{1}+\mu_{2}\right) \\
& =D_{f^{\prime}}(u)
\end{aligned}
$$

and for any vertex $w$ in $I(u, v)$ distinct from $u$ and $v$ :

$$
\begin{array}{r}
D_{f^{\prime}}(w)<D_{f}(w)-d(u, v) \mu_{1} \leq D_{f}(u)-\epsilon_{2}-d(u, v) \mu_{1}= \\
D_{f}(u)-d(u, v)\left(\mu_{1}+\mu_{2}\right)=D_{f^{\prime}}(u)
\end{array}
$$


and for any other vertex $x$ :

$$
\begin{aligned}
D_{f^{\prime}}(x)<D_{f}(x)-(d(u, v)+1) \mu_{1}<D_{f}(z)-\mu_{1}-d(u, v) \mu_{1}= \\
D_{f}(u)-d(u, v) \mu_{1}=D_{f^{\prime}}(u) .
\end{aligned}
$$

This implies that $v$ is the unique antimedian of $f^{\prime}$, while $u$ is a local antimedian, which is not an antimedian vertex. This contradicts the assumption, by which the proof is complete.

Above we established the equivalence of real-valued weight functions, rational-valued weight functions, and signed profiles with respect to medians etcetera. The next theorem is now an easy consequence of the previous results.

Theorem 3.7. Let $G$ be a connected graph. Then the following conditions are equivalent.

(i) The antimedian set $A M(f)$ is connected, for all weight functions $f$ on $G$.

(ii) $A M(f)=A M_{l o c}(f)$, for all weight functions $f$ on $G$.

(iii) The median set $M(f)$ is connected, for all weight functions $f$ on $G$.

(iv) $M(f)=M_{l o c}(f)$, for all weight functions $f$ on $G$.

(v) $A M(f)=A M_{l o c}(f)$, for all rational weight functions $f$ on $G$.

(vi) $A M(\pi)=A M_{l o c}(\pi)$, for all signed profiles $\pi$ on $G$.

(vii) $M(f)=M_{l o c}(f)$, for all rational weight functions $f$ on $G$.

(viii) $M(\pi)=M_{l o c}(\pi)$, for all signed profiles $\pi$ on $G$.

Proof. (i) up to (iv) are equivalent by Proposition 3.6, and Remark 3.1 .

$(i i) \Rightarrow(v)$ follows trivially.

$(v) \Rightarrow($ ii) follows from Lemma 3.5.

$(v) \Rightarrow(v i)$ : Let $\pi$ be a signed profile on $G$. Now consider its associated weight function $f_{\pi}$. By Lemma 3.2, we have $D\left(v, f_{\pi}\right)=D(v, \pi)$. Since $D_{f_{\pi}}$ cannot have any local maximum that is not a global maximum, $D_{\pi}$ also cannot have any local maximum that is not a global maximum.

$(v i) \Rightarrow(v)$ : Let $g$ be any rational weight function on $G$. By Lemma 3.3, there is a positive integer $t$ and a signed profile $\pi$ such that $f_{\pi}=t g$. By Lemma 3.2, $D_{f_{\pi}}=D_{\pi}$, and, as observed above, $D_{f_{\pi}}$ has a local maximum that is not a global maximum if and only if $D_{g}$ have a local maximum that is not a global maximum. So $D_{g}$ cannot have a local maximum that is not a global maximum.

The equivalence of (vii) and (viii) with the other statements follows similarly.

\section{Consensus Strategies}

If one wants to find the median set of a positive profile in a tree, then there exists a simple strategy formulated by Goldman [12] already in 1971. It reads as follows. When at vertex $u$, consider neighbor $v$ of $u$. If there is a majority of the profile closer to $v$ than to $u$, then move to $v$. In [20] this Majority Strategy was formulated for arbitrary graphs. There it was proved that the Majority Strategy produces the median set for any positive profile starting at any vertex if and only if the graph is a median graph, Theorem 4.1 below. For more details, we refer the reader to [20,4]. A connected graph $G$ is called a median graph, if every triple of vertices in $G$ has a unique median. One of the main reasons underlying this result is that the structure of median sets is very nice in median graphs. In [4] four 
other related consensus strategies for positive profiles are studied. Antimedian sets are not so well-structured. So one cannot expect such deep results for signed profiles. But it is still possible to obtain some nice and unexpected results. Below we present a number of consensus strategies for signed profiles similar to the Majority Strategy from [18]. They are analogues of those in [4], but now formulated for signed profiles.

In all the strategies below the input is a connected graph $G$, a profile $\pi$, and an initial vertex at which the strategy starts. There are two possibilities: one gets stuck at a vertex, or it is possible to visit vertices more than once. In the latter case the strategy could get into a loop, so the stopping rule must be more sophisticated here. In all cases, the output after stopping is the single vertex where one gets stuck or the set of vertices visited at least twice. Steps 1, 3 and 4( $i$ ) below are the same for all strategies, so we list these only in the first instance. In all other instances we only list Step 2, describing when one moves to a neighbor, and Step 4(ii), the stopping rule when one does not get stuck.

\section{Majority Strategy}

1. Start at the initial vertex.

2. [MoveMS] If we are in $u$ and $v$ is a neighbor of $u$ with $\left\|\pi_{v u}\right\| \geq \frac{1}{2}\|\pi\|$, then we move to $u$.

3. We move only to a vertex already visited if there is no alternative.

4. We stop when

(i) we are stuck at a vertex $u$ or

(ii) [TwiceMS] we have visited vertices at least twice, and

for each vertex $u$ visited at least twice and each neighbor $v$ of $u$, either $\left\|\pi_{v u}\right\|<\frac{1}{2}\|\pi\|$ or $v$ is also visited at least twice.

Before presenting the other strategies we quote the main theorem from [20]. This theorem has been the motivation for studying such strategies on graphs. It also shows the special role of median graphs within the Class of All Graphs. Due to the structure theory of median graphs, the equivalence of $(i i)$ and $(i i i)$ on median graphs in the theorem is not surprising. But otherwise it would not have been something one would expect at first sight.

Theorem 4.1. [Majority Theorem] Let $G$ be a graph. Then the following conditions are equivalent.

(i) G is a median graph.

(ii) The Majority Strategy produces the median set $M(\pi)$ from any initial vertex, for each positive profile $\pi$ on $G$.

(iii) The Majority Strategy produces the same set from any initial vertex, for each positive profile on $G$.

In the majority strategy one moves towards majority. A slightly different point of view is to move away from minority. This seems to be the same, but it is not, as we will see below. This latter strategy is known as the Condorcet Strategy.

\section{Condorcet Strategy}

2. [MoveCS] If we are in $u$ and $v$ is a neighbor of $u$ with $\left\|\pi_{u v}\right\| \leq \frac{1}{2}\|\pi\|$, then we move to $v$.

4. (ii) [TwiceCS] we have visited vertices at least twice, and for each vertex $u$ visited at least twice and each neighbor $v$ of $u$, either $\left\|\pi_{u v}\right\|>\frac{1}{2}\|\pi\|$ or $v$ is also visited at least twice. 
In non-bipartite graphs the subprofile $\pi_{u}^{v}$ of $\pi$, for an edge $u v$, is not always empty. From the viewpoint of voting, one might say that the elements of $\pi_{u}^{v}$ abstain from voting when the choice is between $u$ and $v$. So these may be ignored when the question is whether to move from $u$ to $v$. This is the idea behind the Plurality Strategy. Note that on bipartite graphs Majority and Plurality coincide.

\section{Plurality Strategy}

2. [MovePS] If we are in $u$ and $v$ is a neighbor of $u$ with $\left\|\pi_{v u}\right\| \geq\left\|\pi_{u v}\right\|$, then we move to $v$.

4. ( ii) [TwicePS] we have visited vertices at least twice, and for each vertex $u$ visited at least twice and each neighbor $v$ of $u$, either $\left\|\pi_{v u}\right\|<\left\|\pi_{u v}\right\|$ or $v$ is also visited at least twice.

The next two strategies were introduced to find a (local) minimum based on a heuristic function in a search graph. They are also known as Hill Climbing and Steepest Ascent Hill Climbing, respectively.

\section{Ascent Strategy}

2. [MoveAS] If we are in $u$ and $v$ is a neighbor of $u$ with $D(v, \pi) \leq D(u, \pi)$, then we move to $v$.

4. (ii) [TwiceAS] we have visited vertices at least twice, and for each vertex $u$ visited at least twice and each neighbor $v$ of $u$, either $D(v, \pi)>D(u, \pi)$ or $v$ is also visited at least twice.

\section{Steepest Ascent Strategy}

2. [MoveSAS] If we are in $u$ and $v$ is a neighbor of $u$ with $D(v, \pi) \leq D(u, \pi)$, and $D(v, \pi)$ is minimum among all neighbors of $u$, then we move to $v$.

\section{4. $($ ii $)[$ TwiceSAS $]=[$ TwiceAS $]$.}

The next simple Lemma is an analogue of Lemma 1 in [4] for signed profiles with the same conclusion. Note that the Plurality and Ascent strategy produce the same output for signed profiles on any connected graph. On bipartite graphs both coincide with Majority.

Lemma 4.2. Let $G$ be a connected graph and $\pi$ a signed profile on $G$. Plurality Strategy makes a move from vertex $v$ to vertex $u$ if and only if $D(u, \pi) \leq D(v, \pi)$.

Proof. The assertion follows immediately from the following computation:

$$
\begin{aligned}
D(v, \pi)- & D(u, \pi)= \\
= & \sum_{x \in \pi_{v u}} s_{x} d(v, x)+\sum_{x \in \pi_{u v}} s_{x} d(v, x)-\sum_{x \in \pi_{v u}} s_{x} d(u, x)-\sum_{x \in \pi_{u v}} s_{x} d(u, x) \\
= & \sum_{x \in \pi_{v u}} s_{x} d(v, x)+\sum_{x \in \pi_{u v}} s_{x} d(v, x)-\sum_{x \in \pi_{v u}} s_{x}(d(v, x)+1)- \\
& \sum_{x \in \pi_{u v}} s_{x}(d(v, x)-1) \\
= & \left\|\pi_{u v}\right\|-\left\|\pi_{v u}\right\| .
\end{aligned}
$$


The various strategies are quite similar. But on general graphs they are all different. We present some examples to show this. The first example shows that Plurality, Condorcet and Majority strategies are pairwise distinct. Consider the profile $\pi=(+a,+b,-c,+d$, $+e,+f)$ on the graph shown in Figure 1. We have $\left\|\pi_{u v}\right\|=1,\left\|\pi_{v u}\right\|=0,\left\|\pi_{v a}\right\|=$ $\left\|\pi_{v b}\right\|=\left\|\pi_{v d}\right\|=\left\|\pi_{v e}\right\|=2\left\|\pi_{v c}\right\|=4,\left\|\pi_{a v}\right\|=\left\|\pi_{b v}\right\|=\left\|\pi_{d v}\right\|=\left\|\pi_{e v}\right\|=1$, $\left\|\pi_{c v}\right\|=-1,\left\|\pi_{u a}\right\|=\left\|\pi_{u b}\right\|=\left\|\pi_{u d}\right\|=\left\|\pi_{u e}\right\|=\left\|\pi_{u f}\right\|=3,\left\|\pi_{u c}\right\|=5,\left\|\pi_{a u}\right\|=$ $\left\|\pi_{b u}\right\|=\left\|\pi_{d u}\right\|=\left\|\pi_{e u}\right\|=\left\|\pi_{f u}\right\|=1,\left\|\pi_{c u}\right\|=-1$.

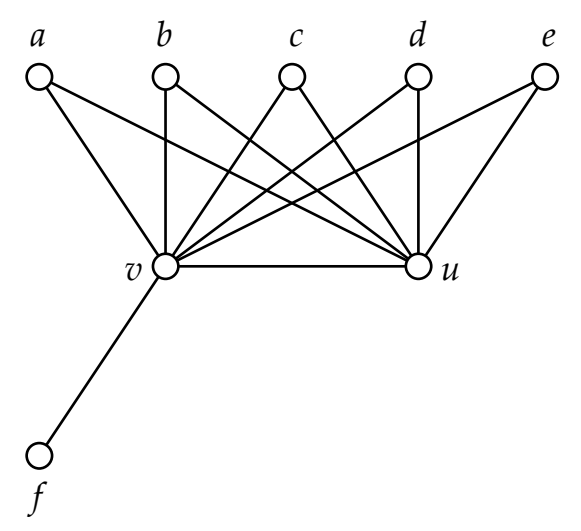

Figure 1: Consensus strategies differing on a graph

Apply all the strategies starting at $u$. Using Majority we may not move to any of its neighbors, so we are stuck at $u$. Thus the outcome of Majority is $\{u\}$. Note that we have $\left\|\pi_{u x}\right\| \leq \frac{1}{2}\|\pi\|$, for any neighbor $x$ of $u$ other than $c$. So, if we use Condorcet, then we can move to any of its neighbors except $c$. Note also that from $a, b, d$ and $e$ a move to either $u$ or $v$ is allowed, but from $v$ we can move only to $u$. Thus using Condorcet we may move along $u, a, b, d, e, v$. Hence the output of the Condorcet Strategy is $\{u, a, b, d, e, v\}$. When we use Plurality, then we can move only to $v$ and we get stuck at $v$. Hence the output of Plurality is $\{v\}$. Ascent and Steepest Ascent strategies also produce the output $\{v\}$.

It is shown in [4] that Steepest Ascent is essentially different from the other strategies for positive profiles. Note that the other strategies might make a move from $u$ as soon as they find a neighbor $v$ of $u$ that satisfies the condition for a move, while Steepest Ascent has to check all neighbors of $u$ before it can make a move. For a comparison of efficiencies of these strategies, see [3].

The following example shows that the first four strategies might not even find the median vertex, even if the graph is bipartite. Consider the complete bipartite graph $K_{2,5}$ with vertices $a, b$ and $1,2,3,4,5$, where two vertices are adjacent if and only if one is a 'letter' and the other is a 'numeral'. Now take the profile $\pi=(+b,+1,+1,+1,+2,+2,+2,+3$, $+3,+3,+4,-5)$. Then we have $D(a, \pi)=11, D(b, \pi)=9, D(4, \pi)=21, D(5, \pi)=17$ and $D(i, \pi)=13$, for $i=1,2,3$. Take 1 as initial vertex and assume that we check its neighbors in alphabetical order. Then Majority, Condorcet, Plurality and Ascent strategies move to $a$ and get stuck there, whereas Steepest Ascent moves to $b$ and thus finds the median vertex of $\pi$.

It was already shown in [4] that Plurality produces the median set for any positive 
profile if and only if all median sets in the graph are connected. Hence Plurality produces the antimedian set for negative profile in such graphs. Moreover the antimedian sets are connected for all negative profiles in such graphs.

In the case of finding antimedian sets one would want to have the "converse" of the above strategies, that is, apply the strategy on $-\pi$ instead of $\pi$. Because we are working with signed profiles these are strategies in their own right. We list them below, with their appropriate names.

\section{Minority Strategy}

Minority applied to $\pi$ is identical with Majority applied to $-\pi$.

\section{Scarcity Strategy}

Scarcity applied to $\pi$ is identical with Plurality applied to $-\pi$.

\section{Descent Strategy}

Descent applied to $\pi$ is identical with Ascent applied to $-\pi$.

\section{Steepest Descent Strategy}

Steepest Descent applied to $\pi$ is identical with Steepest Ascent applied to $-\pi$.

It is interesting to note that Scarcity produces the antimedian set in hypercubes. Recall that the $n$-dimensional hypercube $Q_{n}$, the $n$-cube for short, has the 0,1 -vectors of length $n$ as its vertices, two vertices being adjacent if the corresponding vectors differ in exactly one coordinate. Take any $i$ with $1 \leq i \leq n$. Let $Q_{n, i}^{0}$ be the $(n-1)$-dimensional subcube consisting of the vertices with a 0 as $i$-th coordinate, and let $Q_{n, i}^{1}$ be the complementary subcube consisting of the vertices with a 1 as $i$-th coordinate. For a profile $\pi$, let $\pi_{i}^{0}$ be the subprofile of $\pi$ in $Q_{n, i}^{0}$, and let $\pi_{i}^{1}$ to the subprofile of $\pi$ in $Q_{n, i}^{1}$. Let $W$ be the set of vertices in $Q_{n}$, for which $\pi$ has a signed minority in each coordinate. That is, $x$ lies in $W$ if and only if $x$ has a 0 in the $i$-th coordinate when $\left\|\pi_{i}^{1}\right\|>\left\|\pi_{i}^{0}\right\|$, and a 1 when $\left\|\pi_{i}^{0}\right\|>\left\|\pi_{i}^{1}\right\|$, and a 0 or 1 when $\left\|\pi_{i}^{0}\right\|=\left\|\pi_{i}^{1}\right\|$. This is precisely the antimedian set of $\pi$. It is also a subcube of dimension $d$, where $d$ is the number of coordinates, for which $\left\|\pi_{i}^{0}\right\|=\left\|\pi_{i}^{1}\right\|$.

Proposition 4.3. Scarcity strategy produces the antimedian set on a hypercube for any signed profile.

Proof. Take any $i$ with $1 \leq i \leq n$. Take any vertex $u$ in $Q_{n, i}^{0}$, and let $v$ be its neighbor in $Q_{n, i}^{1}$. Then we have $\pi_{u v}=\pi_{i}^{0}$ and $\pi_{v u}=\pi_{i}^{1}$. So, if $\left\|\pi_{i}^{0}\right\| \geq\left\|\pi_{i}^{1}\right\|$, then we move from $Q_{n, i}^{0}$ to $Q_{n, i}^{1}$. And if $\left\|\pi_{i}^{0}\right\|>\left\|\pi_{i}^{1}\right\|$, then we never move back to $Q_{n, i}^{0}$. So Scarcity moves to the set of vertices $W$ in $Q_{n}$, for which $\pi$ has a signed minority in each coordinate. This is precisely the antimedian set of $\pi$.

In general Scarcity will not always produce an antimedian. For example when we use Scarcity moves in a tree we will always stuck at leaf nodes, as we can see in the following lines. Consider a tree $T$ with at least three leaves, and a positive profile $\pi$ of length $k$ on $T$. Take any leaf $v$ that occurs less than $\frac{1}{2} k$ times in $\pi$, and let $u$ be the neighbor of $v$ in $T$. Note that $d(v, x)=d(u, x)+1$ for any $x \neq v$ in $T$. Obviously we will move to $v$ from $u$, but we will never move back to $v$ using Scarcity. But $v$ need not be the antimedian of $\pi$. If the profile is "close" to $u$, then obviously antimedian will be at some other leaves far away. 
Next we prove an analogue of the main Theorem (Theorem 8 in [4]) for positive profiles in the case of signed profiles.

Theorem 4.4. The following are equivalent for a connected graph $G$.

(i) The Scarcity Strategy produces $A M(\pi)$ from any initial vertex, for all signed profiles $\pi$ on $G$.

(ii) $A M(\pi)$ is connected, for all signed profiles $\pi$ on $G$.

(iii) $A M(\pi)=A M_{l o c}(\pi)$, for all signed profiles $\pi$ on $G$.

(iv) Descent Strategy produces $A M(\pi)$ from any initial vertex, for all signed profiles $\pi$ on G.

$(v)$ Steepest Descent Strategy produces $A M(\pi)$ from any initial vertex, for all signed profiles $\pi$ on $G$.

(vi) Scarcity, Descent, and Steepest Descent Strategy each produce the same set from any initial vertex, for all signed profiles.

Proof. $(i) \Rightarrow(i i)$ : Suppose the antimedian set is not connected for some profile $\pi$. Then let $u$ and $v$ be two vertices in different components of $A M(\pi)$. Now, if Scarcity starts at $u$, then it cannot reach vertex $v$, because a move from an antimedian vertex to a nonantimedian vertex is not possible by Lemma 4.2. So the set computed by Scarcity will not include $u$, which is a contradiction.

$($ ii $) \Rightarrow($ iii $)$ : This follows from Theorem 3.7.

$($ iii $) \Rightarrow(i v)$ : Starting at any vertex, Descent always finds a local maximum. Since this local maximum is also global, it follows that Descent always reaches an antimedian, and since the antimedian set is connected, Descent finds all antimedian vertices.

$(i v) \Rightarrow(i)$ : Assume that Descent finds the antimedian set. This means that Descent will move to an antimedian starting from any vertex and finds all the other antimedians. The same moves will be made by Scarcity, by Lemma 4.2. Hence Scarcity will compute the antimedian set correctly.

$($ iii $) \Rightarrow(v)$ follows similarly as $(i i i) \Rightarrow(i v)$.

$(v) \Rightarrow(i i)$ follows from the fact that Steepest Descent finds a local maximum and does move from antimedian to antimedian but does not move from an antimedian to a non-antimedian.

$(i) \Rightarrow(v i)$ is obvious.

$(v i) \Rightarrow(i)$ follows from the fact that, starting from an antimedian, Scarcity can produce only a set of antimedians which includes the initial vertex. So starting from any vertex it produces the same set if and only if the produced set is actually $A M(\pi)$. The same argument works for Descent and Steepest Descent.

\section{The Majority Strategy for Signed Profiles}

In this section a characterization for hypercubes is obtained as the graphs for which Majority always produces the median set for any signed profile. Before stating the result, we need a few facts from the theory of median graphs as developed in [18]. A median graph is bipartite, and does not contain $K_{2,3}$ as induced subgraph. This implies that any two vertices at distance 2 have either one or two common neighbors. It is proved in [18] that a graph $G$ is a hypercube if and only if it is a median graph in which any two vertices at distance 2 have exactly two common neighbors. For any vertex $w$ in a graph $G$, we write $N_{i}(w)=\{x \mid d(x, w)=i\}$, and $N_{>i}(w)=\bigcup_{j>i} N_{j}(w)$. 
Proposition 5.1. Let $A M$ be the antimedian function on a median graph $G$. Then $A M(\pi)$ is connected for every signed profile $\pi$ if and only if $G$ is a hypercube.

Proof. If $G$ is a hypercube, then Proposition 4.3 gives us the required result.

Conversely, let $G$ be a median graph for which the antimedian set is connected, for any signed profile. Let $u$ and $v$ be two vertices at distance 2, and let $w$ be a common neighbor of $u$ and $v$. Due to the above mentioned characterization of hypercubes in [18] we have to prove that there exists a unique common neighbor of $u$ and $v$ different from $w$. Consider the profile $\pi=(+w)$ of length 1 . Note that, for any $x$ in $N_{j}(w)$, we have $D_{f}(x)=j$. So $N_{>0}(w)=V-w$ is a level set with respect to $\pi$. Due to Proposition3.6 any level set of $\pi$ induces an isometric subgraph. So, within $V-w$, the vertices $u$ and $v$ have distance 2 as well, that is, there is a common neighbor $z$ in $V-x$. Since a median graph is bipartite and does not contain $K_{2,3}$, this neighbor is unique.

The next theorem is an analogue of the majority theorem for signed profiles which turns out to be a new characterization of hypercubes.

Theorem 5.2. A graph $G$ is a hypercube if and only if the Majority Strategy, starting from any initial vertex, produces the median set for any signed profile on $G$.

Proof. If $G$ is a hypercube, then, by Proposition 4.3, Scarcity produces the antimedian set for any signed profile. So, since the hypercube is bipartite, Minority produces the antimedian set for any signed profile, whence Majority produces the median set for any signed profile.

Conversely, assume that Majority produces the median set for any signed profile. Then it also produces the median set for any positive profile. So, by Theorem 4.1 , the graph is a median graph. Hence, by Proposition 5.1, the graph is a hypercube.

\section{Graphs for which Scarcity produces the Antimedian Set for any Signed Profile}

In this section we discuss some graph classes for which Scarcity always produces the antimedian set for any signed profile.

First we consider the Hamming graphs. Let $k_{1}, \ldots, k_{n}$ be positive integers, and let $V$ be the Cartesian product

$$
\Pi_{i=1}^{n}\left\{0,1, \ldots, k_{i}-1\right\} .
$$

The Hamming graph $H_{k_{1}, \ldots, k_{n}}$ is the graph with vertex set $V$, in which two vertices are joined by an edge if and only if the corresponding vectors differ in exactly one coordinate. The properties for Hamming graphs needed here probably all belong now to folklore, but could also be found in $[18,19]$, where they were characterized for the first time. The set of vertices in $H=H_{k_{1}, \ldots, k_{n}}$ having $a$ in the $i$-th position of the corresponding vector is denoted as $H_{k_{1}, \ldots, k_{n}, i}^{a}$, or simply as $H_{i}^{a}$. For a profile $\pi$, we denote its subprofile contained in $H_{i}^{a}$ by $\pi_{i}^{a}$.

Let $\pi$ be a profile on $H=H_{k_{1}, \ldots, k_{n}}$. Fix a position $i$, for which $k_{i} \geq 2$, and let $a$ and $b$ be distinct elements in $\left\{0, \ldots, k_{i}-1\right\}$. Let $u$ be a vertex in $H_{i}^{a}$, and let $v$ be its neighbor in $H_{i}^{b}$. Then $\pi_{u v}=\pi_{i}^{a}$ and $\pi_{v u}=\pi_{i}^{b}$. Note that, if $u$ is in $A M(\pi)$, then we have $\left\|\pi_{i}^{a}\right\| \leq\left\|\pi_{i}^{b}\right\|$. This holds for every $b$ in $\left\{0, \ldots, k_{i}-1\right\}$ distinct from $a$. In this case we 
say that there is a signed minority at $a$ in position $i$. Clearly, an antimedian vertex in $H$ is a vertex with a signed minority in each coordinate. Let $m_{i}$ be the number of elements in $\left\{0, \ldots, k_{i}-1\right\}$ with a signed minority, for $i=1, \ldots, n$. Then the antimedian set of $\pi$ induces a subgraph isomorphic to $H_{m_{1}, \ldots, m_{n}}$. Obviously, any antimedian set is connected.

Proposition 6.1. Starting form any vertex Scarcity Strategy produces the antimedian set on a Hamming graph for any signed profile.

Proof. Let $\pi$ be a (signed) profile on the Hamming graph $H=H_{k_{1}, \ldots, k_{n}}$. Take any $i$ with $1 \leq i \leq n$ and any vertex $u$ in $H_{i}^{a}$, and let $v$ be its neighbor in $H_{i}^{b}$ with $b \neq a$. If $\left\|\pi_{i}^{a}\right\| \geq\left\|\pi_{i}^{b}\right\|$, then we move from $H_{i}^{a}$ to $H_{i}^{b}$. And if $\left\|\pi_{i}^{a}\right\|>\left\|\pi_{i}^{b}\right\|$, then we never move back to $H_{i}^{a}$. So Scarcity moves to the set of vertices $W$ in $H$ for which $\pi$ has a signed minority in each coordinate. This is precisely the antimedian set of $\pi$.

Graphs which admit a scale- $\lambda,(\lambda \geq 2)$ embedding into a hypercube is called an $\ell_{1}$ graph. The Johnson graphs and half cubes are important classes of $\ell_{1}$ graphs which occur as hosts for isometric embeddings of graphs, [8]. Next we consider the Johnson graphs followed by half cubes. The Johnson graph $J_{n, k}$ has as vertices the $k$-element subsets of $\{1,2, \ldots, n\}$, and two vertices are adjacent if and only if their intersection has size $k-1$. In other words the vertices 'differ' in exactly one element. Some special Johnson graphs are: $J_{n, 1}$ is the complete graph on $n$ vertices, $J_{n, 2}$ is the $n$-triangular graph, and $J_{n, 3}$ is $n$-tetrahedral graph. Since each vertex $u$ in $J_{n, k}$ corresponds to a $k$-element subset $X$ of $\{1,2, \ldots, n\}$, we represent $u$ with the vector $\left[u_{1}, \ldots, u_{n}\right]$, where

$$
u_{i}= \begin{cases}1 ; & i \in X \\ 0 ; & i \notin X\end{cases}
$$

Clearly the total number of 1's in each vector representation is $k$. Moreover adjacent vertices differ in two positions. Note that mapping these vectors to the corresponding vectors in a hypercube $Q_{n}$ corresponds to a so-called scale- 2 embedding, that is, two vertices at distance $d$ in the Johnson graph are mapped onto vertices at distance $2 d$ in the hypercube, for any two vertices. Since below the antimedian sets in more than one graph will be considered, we denote the antimedian set of $\pi$ in $G$ also by $A M(\pi, G)$, and so forth.

Proposition 6.2. Let $G$ be a Johnson graph. Then $M(\pi)$ and $A M(\pi)$ are also Johnson graphs.

Proof. Assume that $G=J(n, k)$. Consider the scale-2 embedding of $G$ in to the hypercube $Q_{n}$. Let $\pi$ be a profile in $G$, and let $M\left(\pi, Q_{n}\right)$ be isomorphic to $Q_{r}$. Without loss of generality we may assume that, for all the vertices $u=\left[u_{1}, \ldots, u_{n}\right]$ in this subcube, the coordinates at positions $r+1$ up to $n$ are all the same, and that in the remaining positions $1, \ldots, r$ values 0 and 1 are taken. Let $m$ be the total number of 1 's, in positions $r+1, \ldots, n$.

We analyze the properties of median sets in $G$ by considering two cases.

Case 1. $M\left(\pi, Q_{n}\right) \cap G \neq \emptyset$.

Clearly $M(\pi, G)$ induces a subgraph isomorphic to $J_{r,(k-m)}$.

Case 2. $M\left(\pi, Q_{n}\right) \cap G=\emptyset$.

In this case we have either $m<k-r$ or $m>k$. Clearly, if $m<k-r$, we get a vertex in $G$ by changing a minimum number of coordinates, say $p$, from the vertex in $M\left(\pi, Q_{n}\right)$ having $1 \mathrm{~s}$ in positions $1, \ldots, r$. 
Similarly, when $m>k$, we get a vertex in $G$ with a minimum number of changes, by selecting the vertex with 0 's in positions $1, \ldots, r$. Since we are looking for a median set in $G$, we select the positions, in such a way that the change in remoteness is minimum. Thus we select $p$ coordinate positions with smaller signed majority values. If the signed majority values are distinct we get a single vertex in $G$. Otherwise we make a selection among, say $p^{\prime}$ positions. In this case the subgraph induced by the vertices of $G$ thus obtained will be isomorphic to $J_{p^{\prime}, p}$.

Since the remoteness is same for all vertices in the median set we get the same result independent of the vertex selected. Hence we get a subgraph that is a Johnson graph as the median set.

With similar arguments, by taking the signed minority values at coordinate positions, we can prove that antimedian sets also induce some Johnson graph. This completes the proof.

From the above theorem we have the following corollary.

Corollary 6.3. Let $G$ be a Johnson graph. Then $M(\pi)$ and $A M(\pi)$ are connected, for any signed profile $\pi$ in $G$.

From the above Corollary and Theorem 4.4 we have:

Corollary 6.4. Starting from any vertex on a Johnson graph Scarcity strategy produces the antimedian set for any signed profile.

Next, we consider halfcubes. The vertex set of a halfcube is the subset of the vertices of the hypercube $Q_{n}$ with an even (respectively, odd) number of ones in their vector representation. Two vertices are adjacent when they differ in exactly two positions, see [8]. Halfcubes also admit a scale- 2 embedding into the corresponding hypercube.

Theorem 6.5. Let $G$ be a halfcube, then $M(\pi, G)$ and $A M(\pi, G)$ are connected for any signed profile $\pi$ in $G$.

Proof. Let $Q_{n}$ be the hypercube of dimension $n$ in which $G$ is scale- 2 embedded. Let $\pi$ be an arbitrary profile in $G$ and $\|\pi\|=k$. Note that by applying the Majority rule for the given profile $\pi$ of the halfcube embedded into hypercube $Q_{n}$ (looking as the vertices of a hypercube), we get the median of $\pi$ in $Q_{n}$ which will be a sub-hypercube, say $Q_{r}$. We analyze the property of $M(\pi, G)$ by considering the following two cases separately.

Case 1. $M\left(\pi, Q_{n}\right)$ is a hypercube $Q_{r}$ of dimension at least one.

Clearly $Q_{r}$ has half vertices in the corresponding halfcube - call this set $X$. Set $X$ forms a halfcube in $G$, hence $X$ is connected. Since the graph $G$ is scale- 2 embedded the remoteness in $G$ is obtained by dividing the corresponding remoteness in $Q_{n}$ by 2, we get $M(\pi, G)=X$, as we follow the signed Majority rule on $\pi$.

Case 2. $M\left(\pi, Q_{n}\right)$ in $Q_{n}$ contains exactly one vertex say $x$.

If $x$ belongs to $G$, then clearly $M(\pi, G)=\{x\}$ as the case may be and hence we are done.

So assume that $x$ is not in $G$. Note that $x=\left(x^{1}, \ldots, x^{d}\right)$ can be obtained from the signed Majority rule among coordinates of the profile $\pi$. Let $m_{i}, 1 \leq m_{i} \leq n$ be the signed majority at each position. Let $m=\min \left\{m_{1}, \ldots, m_{n}\right\}$. Clearly if for any vertex $y$ obtained by changing any single $i^{t h}$ coordinate of $x$, the remoteness changes by $2 m_{i}-k$, where $\|\pi\|=k$. This change in remoteness is minimum for coordinates having signed 
Majority value $m$. Hence $M(\pi, G)$ is precisely the set of vertices obtained from $G$ by changing any coordinate of $x$, having minimum signed Majority $m_{i}$. These vertices are all adjacent to $x$, and hence forms a clique in $G$. Thus $M(\pi, G)$ is connected for any signed profile.

With similar arguments and by taking $m$ as maximum $\left(m_{1}, \ldots, m_{n}\right)$, where each $m_{i}$ is signed minority, we can prove that $A M(\pi, G)$ is also connected for any profile, which completes the proof.

From the proof of the above theorem, we have the following corollary.

Corollary 6.6. Let $G$ be a halfcube, then $M(\pi, G)$ and $A M(\pi, G)$ induce a halfcube in $G$ or a clique, for any profile $\pi$ in $G$.

From Theorem 6.5 and Theorem 4.4 we have:

Corollary 6.7. Starting from any arbitrary vertex in a halfcube Scarcity Strategy always produce antimedian set for any signed profile

\section{Concluding remarks}

In this paper, we have proved that the classes of graphs in which the consensus strategies Scarcity, Descent and Steepest Descent will always produce the antimedians for any arbitrary signed profile is precisely the class of graphs with connected antimedians. This class of graphs is characterized in terms of (local) medians and (local) antimedians of (rational) weight functions. Also, we proved that, among the median graphs, the hypercubes are precisely the graphs with connected antimedians for an arbitrary signed profile. Moreover, we presented some classes on which Scarcity produces the antimedian set for any signed profile. An intriguing question remains: Which classes of graphs have connected antimedians for arbitrary signed profiles?

\section{References}

[1] K. Balakrishnan, B. Brešar, M. Changat, S. Klavžar, M. Kovše and A. R. Subhamathi, On the remoteness function in median graphs, Discrete Appl. Math. 157 (2009), 3679-3688.

[2] K. Balakrishnan, B. Brešar, M. Changat, S. Klavžar, M. Kovše and A. R. Subhamathi, Simultaneous embeddings of graphs as median and antimedian subgraphs, Networks $\mathbf{5 6}$ (2010), 90-94.

[3] K. Balakrishnan, M. Changat and H. M. Mulder, Median computation in graphs using consensus strategies, Report EI 2006, Econometrisch Instituut, Erasmus Universiteit, 2006, Rotterdam.

[4] K. Balakrishnan, M. Changat and H. M. Mulder, The plurality strategy on graphs, Australsian J. Combin. 46 (2010), 191-202.

[5] K. Balakrishnan, M. Changat, I. Peterin, S. Špacapan, P. Šparl and A. R. Subhamathi, Strongly distance-balanced graphs and graph products, Eur. J. Combin. 30 (2009), 1048-1053.

[6] H. J. Bandelt and V. Chepoi, Graphs with connected medians, SIAM J. Discr. Math. 15 (2002), 268-282.

[7] J. P. Barthelemy and B. Monjardet, The median procedure in cluster analysis and social choice theory, Math. Social Sci. 1 (1981), 235-268. 
[8] A. E. Brouwer, A. M. Cohen and A. Neumaier, Distance-Regular Graphs, Springer-Verlag, Berlin, 1989.

[9] P. Cappanera, A survey on obnoxious facility location problems, Technical Report TR-99-11, University of Pisa, 1999.

[10] P. Cappanera, G. Gallo and F. Maffioli, Discrete facility location and routing of obnoxious activities, Discrete Appl. Math. 133 (2003), 3-28.

[11] R. L. Church and R. S. Garfinkel, Locating an obnoxious facility on a network, Transportation science 12 (1978), 107-118.

[12] A. J. Goldman, Optimal center location in simple networks, Transportation Science 5 (1971), 212-221.

[13] S. Klavžar and H. M. Mulder, Median graphs: characterizations, location theory and related structures, J. Combin. Math. Combin. Comput. 30 (1999), 103-127.

[14] F. R. McMorris, H. M. Mulder and F. S. Roberts, The median procdure on median graphs, Discrete Appl. Math. 84 (1998), 165-181.

[15] F. R. McMorris, H. M. Mulder and R. V. Vohra, Axiomatic characterization of location functions, in: H. Kaul and H. M. Mulder (eds.), Advances in interdisciplinary discrete applied mathematcis, Interdisplinary Mathematical Sciences, Vol. 11, World Scientific, Singapore, 2010, pp. 71-91.

[16] F. R. McMorris and R. C. Powers, The median procedure in a formal theory of consensus, Siam J. Discrete Math. 8 (1995), 507-516.

[17] P. B. Mirchandani and R.L. Francis, Discrete Location Theory, Wiley-Interscience, New York, 1990.

[18] H. M. Mulder, The Interval Function of a Graph, Mathematical Centre Tracts 132, Mathematisch Centrum, Amsterdam, 1980.

[19] H.M. Mulder, Interval-regular graphs, Discrete Math. 41 (1982), 253-269.

[20] H. M. Mulder, The Majority Strategy on graphs, Discrete Appl. Math. 80 (1997), 97-105.

[21] H.M. Mulder, Median graphs, A structure theory, in: H. Kaul and H.M. Mulder (eds.), Advances in interdisciplinary discrete applied mathematcis, Interdisplinary Mathematical Sciences, Vol. 11, World Scientific, Singapore, 2010, pp. 93-125.

[22] R. C. Powers, Consensus centered at majority rule, in: H. Kaul and H.M. Mulder (eds.), Advances in interdisciplinary discrete applied mathematcis, Interdisplinary Mathematical Sciences, Vol. 11, World Scientific, Singapore, 2010, pp. 149-166.

[23] R. C. Tansel, R. I. Francis and T. J. Lowe, Location on networks: A survey I, Management Sci. 29 (1983), 482-497.

[24] R. C. Tansel, R. I. Francis and T. J. Lowe, Location on networks: A survey II, Management Sci. 29 (1983), 498-511. 